\title{
Evaluation of Sensitivity and Resistance Risk of Corynespora cassiicola to Isopyrazam and Mefentrifluconazole
}

\author{
Dicheng Ma, ${ }^{1}$ Jiangong Jiang, ${ }^{1}$ Jiamei Zhu, ${ }^{1}$ Lingyan Zhang, ${ }^{1}$ Beixing Li, ${ }^{1}$ Wei Mu, ${ }^{1,2}$ and Feng Liu ${ }^{1,2, \dagger}$ \\ ${ }^{1}$ Shandong Provincial Key Laboratory for the Biology of Vegetable Diseases and Insect Pests, College of Plant Protection, Shan- \\ dong Agricultural University, 61 Daizong Street, Tai' an, Shandong 271018, People's Republic of China \\ ${ }^{2}$ Key Laboratory of Pesticide Toxicology and Application Technique, Shandong Agricultural University, Tai' an, Shandong \\ 271018, China
}

\begin{abstract}
Necrotic lesions on leaves caused by Corynespora leaf spot (CLS) seriously threaten the quality and yield of cucumber in China. Corynespora cassiicola has developed different degrees of sensitivity to various fungicides due to its long-term and extensive application. In our work, the effect of isopyrazam and mefentrifluconazole on different life stages of C. cassiicola was examined. To determine the optimal effect of binary mixtures of isopyrazam and mefentrifluconazole, the two fungicides were mixed at different proportions. Furthermore, the disease suppression of isopyrazam, mefentrifluconazole, and their compound mixture against CLS was evaluated in greenhouse experiments. Ultraviolet (UV) mutagenesis and fungicide-selection methods were per-

germ tube elongation. According to Wadley's and cotoxicity coefficient methods, the optimal proportion of the two-component mixture of isopyrazam and mefentrifluconazole was 1:1. Isopyrazam, mefentrifluconazole, and their binary mixture at 1:1 reduced the disease severity of CLS on potted cucumber plants, with protective effects of 31.11, 24.65 , and $42.12 \%$ and curative effect of $33.90,37.48$, and $42.84 \%$, respectively. Compared with isopyrazam or mefentrifluconazole alone, the binary mixture of the two fungicides at 1:1 did not exert significant influence on the change of $C$. cassiicola sensitivity. Undoubtedly, such data will greatly facilitate the screening of new fungicides for CLS and resistance management.
\end{abstract} formed to assess the risk of resistance development. Among the three life stages tested, isopyrazam showed the weakest inhibition on mycelial growth, and mefentrifluconazole showed the strongest inhibition of
Keywords: Corynespora cassiicola, isopyrazam, mefentrifluconazole, binary mixture, resistance risk assessment
Cucumber (Cucumis sativus L.) is an economically important vegetable crop (de Albuquerque et al. 2016) that can be infected by many fungal pathogens during its growth. Typically, Corynespora cassiicola (Berk. \& M. A. Curtis) C. T. Wei, the causal agent of Corynespora leaf spot (CLS), is considered to be one of the three main pathogens that infect cucumbers, in addition to downy mildew and powdery mildew (Ishii et al. 2007). C. cassiicola, one of the most common species in the genus Corynespora, can attack more than 500 commercial plants from 380 genera, including tomato, soybean, papaya, and rubber, resulting in significant economic losses (Dixon et al. 2009; Kingsland 1986). Massive defoliation of the host caused by CLS severely impairs the quality and yield of cucumber (Ishii et al. 2011). Both high humidity and high temperature $\left(25\right.$ to $\left.35^{\circ} \mathrm{C}\right)$ contribute to the occurrence and spread of CLS (Li et al. 2012); therefore, ordinary plastic greenhouses, which are commonly used for growing cucumber in northern China, are particularly suitable for the occurrence of this disease. Under suitable

${ }^{\dagger}$ Corresponding author: F. Liu; fliu@ @sdau.edu.cn

Funding: This work was supported by the National Key Research Development Program of China (2016YFD0200500) and Provincial Key Research and Development Program of Shandong (2017CXGC0207).

https://doi.org/10.1094/PDIS-02-20-0384-RE

*The $\boldsymbol{e}$-Xtra logo stands for "electronic extra" and indicates that there are supplementary materials published online.

The author(s) declare no conflict of interest.

Accepted for publication 27 May 2020.

C 2020 The American Phytopathological Society environmental conditions, the incidence of the disease is 10 to $25 \%$ and as high as 60 to $70 \%$ or even $100 \%$ in severe cases (Yu et al. 2014).

CLS has been regarded as a minor disease for the past several decades; however, it has attracted considerable attention due to various factors, including the lack of satisfactory control measures and high genetic variability of isolates ( $\mathrm{Li}$ et al. 2012). Recently, CLS has been prevalent in major vegetable-producing areas of Hainan, Yunnan, Henan, Jilin, Liaoning, Beijing, Hebei, and Shandong provinces of China, and CLS is aggravated each year (Zhang et al. 2014). Foliar application of systemic fungicides has played the greatest role in preventing the spread of CLS, even if multiple agricultural measures were integrated to protect host plants from the primary infection of C. cassiicola (De Wolf and Isard 2007; Li et al. 2012). Due to heavy applications of fungicides over time, the pathogen has developed varying degrees of sensitivity to a variety of site-specific fungicides, such as succinate dehydrogenase inhibitors (SDHIs), benzimidazoles, $N$-phenylcarbamates, quinone outside inhibitors (QoIs), and dicarboximides (Date et al. 2004; Ishii et al. 2007; Miyamoto et al. 2009). It is well known that $C$. cassiicola has been rated as a typical fungus with a high risk of resistance development by FRAC (2019). Currently, only eight fungicides have been registered for the management of CLS in China; all of these fungicides are mixed formulations (http://www.chinapesticide.org.cn/). Two or more fungicides can be mixed to improve their inhibition of a pathogen; for example, propiconazole and chitosan-copper complex showed a good synergetic effect against all tested mold fungi, which occur on bamboo (Phyllostachys pubescens Mazel ex H. de Lehaie) (Sun et al. 2012). In another example, the best synergistic effect was observed in the binary mixture of pyraclostrobin and chlorothalonil at 1:7 with a synergistic ratio of 5.8058 (Luo et al. 2016). However, current research has focused on screening and identifying individual new fungicides that manage CLS and verifying their disease suppression. Accordingly, research on the mixed application of two fungicides with different modes of 
action (MOA) requires the use of different models to determine the optimal ratio of the binary mixtures, and whether the fungicide compounds can delay the occurrence of resistance is invaluable.

Isopyrazam (trade name: Reflect; FRAC code 7) is the first product from the pyrazole carboxamide group of SDHI fungicides to be marketed by Syngenta (Basel, Switzerland) (Harp et al. 2011). SDHI fungicides affect the energy metabolism of pathogens by targeting protein complex II on the mitochondrial electron transport chain (Gudmestad et al. 2013). As a broad-spectrum and highly effective fungicide, isopyrazam has exhibited good inhibitory activity against the plant fungi Mycosphaerella graminicola and Botrytis cinerea (Fraaije et al. 2012; Song et al. 2016). Mefentrifluconazole (trade name: Revysol; FRAC code 3) is the latest sterol demethylation inhibitor (DMI) fungicide discovered and developed by BASF (Ludwigshafen, Germany), and mefentrifluconazole was the first new triazole fungicide to come to market after the launch of simeconazole (Zhang et al. 2019). DMI fungicides are known to inhibit cell growth by blocking ergosterol biosynthesis and eventually causing cell membranes to collapse (Koller and Scheinpflug 1987). Unlike other fungicides in the same chemical group, mefentrifluconazole not only has higher activity, but also better environmental friendliness, considering its lower toxicity to mammals and bees (Zhang et al. 2019). The chemical can be used for more than 60 crops worldwide, such as field crops, economic crops, and special crops (Zhang et al. 2019). Furthermore, isopyrazam and mefentrifluconazole have not been registered to defend against CLS in China so far (http:// www.chinapesticide.org.cn/).

In summary, the present study set out to (i) test the bioactivity of isopyrazam and mefentrifluconazole against $C$. cassiicola on the basis of germ tube elongation, spore germination, and mycelial growth; (ii) determine the optimal ratio of isopyrazam and mefentrifluconazole in binary mixtures with different methods; (iii) assess whether the combination of isopyrazam and mefentrifluconazole can enhance their in vivo and in vitro inhibitory effect against CLS; and (iv) evaluate whether the mixed use of isopyrazam and mefentrifluconazole can delay resistance development.

\section{Materials and Methods}

C. cassiicola isolate collection. In 2018, detached cucumber leaves with disease symptoms typical of infection by $C$. cassiicola were obtained from eight major commercial cucumber-producing regions in Shandong, where CLS commonly occurred (Supplementary Material). Small pieces of tissue cut from leaf lesions were first surface sterilized with ethanol (75\%) for $3 \mathrm{~min}$, washed three times with sterilized deionized water, and transferred to potato dextrose agar (PDA; $1,000 \mathrm{ml}$ of deionized water, $20 \mathrm{~g}$ of agar, $20 \mathrm{~g}$ of glucose, and $200 \mathrm{~g}$ of potato) plates, which were amended with streptomycin sulfate to inhibit bacterial growth. Before tests, the $C$. cassiicola isolates were kept on PDA plates in the dark at $25^{\circ} \mathrm{C}$ after single-spore isolation.

Fungicides and chemicals. Technical-grade isopyrazam (99\% active ingredient [a.i.]), kindly supplied by Syngenta Crop Protection Incorporated, and mefentrifluconazole ( $98.7 \%$ a.i.), provided by Beijing Century Aoke Biotechnology Co., Ltd., were used in in vitro fungicide sensitivity assays. Isopyrazam and mefentrifluconazole were dissolved in acetone to obtain stock solutions of $1 \times 10^{4} \mu \mathrm{g} /$ $\mathrm{ml}$ and then stored in the dark at $4{ }^{\circ} \mathrm{C}$ until use. Commercial isopyrazam (125 g/liter emulsifiable concentrate [EC]) and mefentrifluconazole (100 g/liter EC) fungicide formulations were used in in vivo inoculation experiments, and they needed to be diluted with sterile water to working concentration before the experiments.

Sensitivity of $C$. cassiicola isolates to isopyrazam and mefentrifluconazole. In total, 10 isolates were successfully isolated and employed to test the inhibitory effects of isopyrazam and mefentrifluconazole on $C$. cassiicola based on three different life stages. The sensitivity of the mycelial growth of $C$. cassiicola to isopyrazam and mefentrifluconazole was tested on PDA medium. The ability of isopyrazam to inhibit the conidia germination and germ tube elongation of C. cassiicola was estimated on yeast-bacto peptone-acetate (YBA; $10 \mathrm{~g}$ of bacto peptone, $10 \mathrm{~g}$ of yeast extract, $15 \mathrm{~g}$ of agar, $20 \mathrm{~g}$ of sodium acetate, and 1,000 $\mathrm{ml}$ of deionized water) medium, and the inhibition of mefentrifluconazole was tested on water agar (WA; $1,000 \mathrm{ml}$ of deionized water and $20 \mathrm{~g}$ of agar) medium (He at al. 2018; Miyamoto et al. 2010).

For mycelial growth, agar plugs $(7 \mathrm{~mm})$, which were precultured on PDA plates for 6 days in the dark at $25^{\circ} \mathrm{C}$, were placed upside down in the center of a series of Petri dishes $(9 \mathrm{~cm})$ containing media amended with isopyrazam or mefentrifluconazole. After the preliminary experiment, we finally adjusted the concentration of isopyrazam in the medium to $0,0.78125,1.5625,3.125,6.25$, and $12.5 \mu \mathrm{g} / \mathrm{ml}$, and mefentrifluconazole to $0,3.125,6.25,12.5,25$, and $50 \mu \mathrm{g} / \mathrm{ml}$. The final concentration of acetone in the media was adjusted to less than $1 \%$, and the same concentration of acetone was added to the negative control. Four days after cultivation at $25^{\circ} \mathrm{C}$ in the dark, the mean radial growth of each isolate was measured to calculate the inhibition rate. Three independent experiments were conducted, each with two replicates of each isolate-fungicide concentration combination.

Spore suspensions of $1 \times 10^{5}$ spores $/ \mathrm{ml}$ were obtained to measure conidia germination and germ tube elongation (Miyamoto et al. 2009). Briefly, mycelial discs were grown on PDA plates for 7 days in the dark at $25^{\circ} \mathrm{C}$; thereafter, the mycelia were flattened with a glass rod to induce spore production. Aliquots of spore suspension $(100 \mu \mathrm{l})$ were dispersed on YBA (or WA) plates amended with isopyrazam or mefentrifluconazole and then incubated for $8 \mathrm{~h}$ in the dark at $25^{\circ} \mathrm{C}$. Considering that the same fungicide may have different inhibitory effects on $C$. cassiicola at different life stages, after the preliminary experiment, the final concentration of isopyrazam in YBA medium was adjusted to $0,0.75,1.5,3,6$, and $12 \mu \mathrm{g} / \mathrm{ml}$, while the final concentration of mefentrifluconazole in WA medium was adjusted to $0,1.25,2.5,5,10$, and $20 \mu \mathrm{g} / \mathrm{ml}$. We examined germ tube elongation and conidial germination under an optical microscope at $40 \times$ magnification, and spores were considered germinated when the length of the germ tube reached half the length of the spore. The germination rate and germ tube length of 100 spores were examined per plate. The experiment was repeated at three times.

Determination of the optimal proportion of isopyrazam and mefentrifluconazole binary mixtures. In order to give consideration to the effective concentration range of the two fungicides on C. cassiicola and facilitate the preparation of the working solution and concentration calculation, we designed the final concentration of the two-component mixture of isopyrazam and mefentrifluconazole as $0.08,0.4,2,10$, and $50 \mathrm{~g} / \mathrm{ml}$. First, stock solutions of isopyrazam and mefentrifluconazole was diluted separately with sterile water to yield concentrations of $0.8,4,20,100$, and $500 \mu \mathrm{g} / \mathrm{ml}$. Subsequently, two fungicides with the same concentration were mixed in the following proportions: 1:9, 1:4, 3:7, 2:3, 1:1, 3:2, 7:3, 4:1, and 9:1 (isopyrazam/mefentrifluconazole). Mixed fungicide solution was then diluted 10 times with PDA medium, and the sensitivity of $C$. cassiicola to the different binary mixtures of isopyrazam and mefentrifluconazole was determined according to the mycelial growth rate method elaborated above. Isolate $\mathrm{JN}-1$ with moderate sensitivity to isopyrazam and mefentrifluconazole was selected from our isolate collection to determine the optimal proportion of binary mixtures. We input the fungicide concentration and corresponding inhibition rate into the data analysis software DPS to fit regression equation to obtain the $\mathrm{EC}_{50}$ (Tang and Zhang 2012).

The synergistic ratio (SR) was calculated with the Wadley method according to the $\mathrm{EC}_{50}$ values of isopyrazam and mefentrifluconazole and their proportions in the mixtures (Wadley 1967). SR $>1.5$ was considered a synergistic effect, $0.5<\mathrm{SR}<1.5$ was an additive effect, and $\mathrm{SR}<0.5$ was an antagonistic effect.

$\mathrm{EC}_{50}($ Theoretical value $)=1 /($ The proportion of isopyrazam $/$ The $\mathrm{EC}_{50}$ of isopyrazam + The proportion of mefentrifluconazole/ The $\mathrm{EC}_{50}$ of mefentrifluconazole)

$$
\mathrm{SR}=\mathrm{EC}_{50}(\text { Theoretical value }) / \mathrm{EC}_{50}(\text { Actual observed value })
$$

The cotoxicity coefficient (CTC) method developed previously was run to evaluate the bioactivity of the fungicide mixtures (Sun 
and Johnson 1960), where CTC values $>120$ were considered synergistic, CTC values from 80 to 120 were considered additive, and CTC values $<80$ were antagonistic

Toxicity index $(\mathrm{TI})=\mathrm{The}^{\mathrm{EC}} \mathrm{C}_{50}$ of The standard fungicide / The $\mathrm{EC}_{50}$ of tested fungicide

The standard fungicide may be either isopyrazam or mefentrifluconazole, and the former was selected in the present study.

$$
\begin{gathered}
\text { Actual toxicity index }(\mathrm{ATI})=\text { The } \mathrm{EC}_{50} \text { of isopyrazam / } \\
\text { The } \mathrm{EC}_{50} \text { of compound fungicide }
\end{gathered}
$$

Theoretical toxicity index $(\mathrm{TTI})=$ The TI of isopyrazam $\times$ its proportion in compound fungicide +

The TI of mefentrifluconazole $x$

its proportion in compound fungicide

$$
\mathrm{CTC}=\mathrm{ATI} / \mathrm{TTI} \times 100
$$

After the inverse sine transformation, the relationship between the percentage of isopyrazam in the mixture $(\mathrm{K})$ and CTC $(\mathrm{Y})$ was fitted using Origin (OriginLab Corporation, U.S.A.).

$$
\mathrm{X}=\arcsin (\mathrm{K})^{1 / 2}
$$

In vitro bioactivity of the optimum proportion of isopyrazam and mefentrifluconazole against $\boldsymbol{C}$. cassiicola. As mentioned above, we combined several different evaluation methods and

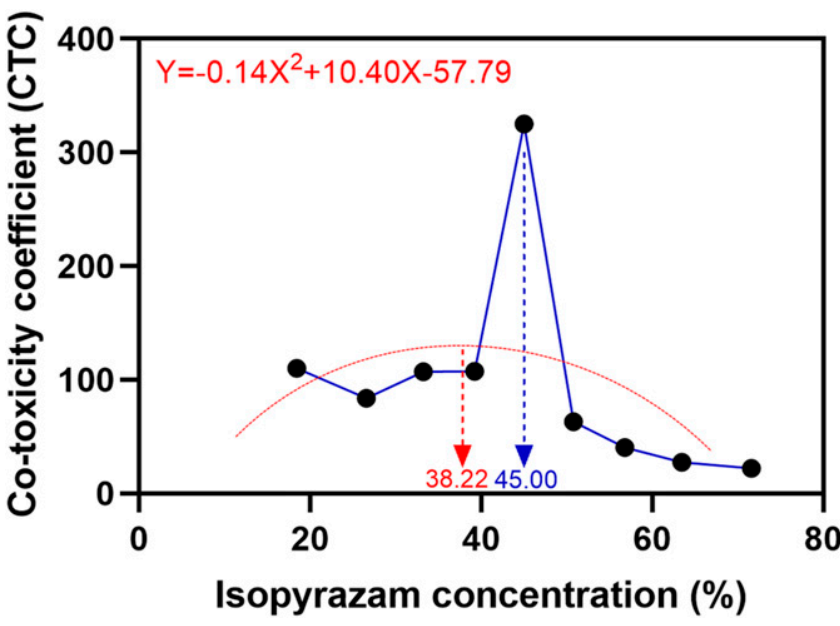

Fig. 1. Determination of the optimal proportion of isopyrazam and mefentrifluconazole binary mixtures. Before data fitting, the percent of isopyrazam in the mixture was converted to the arcsine conversion value. The darker (blue in print) curve indicates the relationship between the percent of isopyrazam in binary mixtures and cotoxicity coefficient, and lighter (red in print) curve indicates their fitting curve. The theoretical maximum value of the cotoxicity coefficient (CTC) was 140.38 (X = $38.22, \mathrm{~K}=38.27 \%$ ), and the measured maximum CTC value was 325.17 ( $\mathrm{X}=$ $45.00, K=50.00 \%$ ). determined that the synergism was most pronounced when isopyrazam and mefentrifluconazole were used in a 1:1 mixture (Fig. 1, Table 1). Next, we determined the sensitivity of the remaining nine C. cassiicola isolates to the optimal mixture according to mycelial growth and evaluated the corresponding synergistic effect.

In vivo bioactivity of the optimum proportion of isopyrazam and mefentrifluconazole against $\boldsymbol{C}$. cassiicola. In greenhouse inoculation experiments, we planted the cucumber cultivar Qiande-2 (Shanghai Qiande Seed Industry Co., Ltd., Shanghai, China) following conventional agronomic practices until the second true leaf grew. In total, four different treatments were arranged for greenhouse experiments: (1-3) isopyrazam, mefentrifluconazole, or iso \& mef (1:1) applied at a concentration of $100 \mu \mathrm{g}$ a.i./ml; and (4) water control. Foliar sprays were applied until runoff with a handheld sprayer. The experiment was repeated three times.

C. cassiicola isolate $\mathrm{JN}-1$ with normal pathogenicity on cucumber was selected from our isolate collection for in vivo inoculation experiment. The spore suspension $\left(1 \times 10^{5}\right.$ spores $\left./ \mathrm{ml}\right)$ of $\mathrm{JN}-1$ was obtained in advance to supply inoculum (Miyamoto et al. 2009). To assess protective effects, potted cucumber plants were artificially inoculated with a spore suspension of JN-1 $24 \mathrm{~h}$ after foliar fungicide application. To assess the curative ability of the fungicide compounds, inoculation was performed $24 \mathrm{~h}$ before the spraying was completed. For successful infection, the leaves of potted cucumber plants were prepunctured by a sterilized needle to create a wound in the center of the leaf near the main vein. We prepared 15 leaves for each treatment to calculate the disease index, and each leaf was inoculated with a pipette of $0.05 \mathrm{ml}$ of spore suspension. After treatment, all potted plants were transferred to a moist artificial climate chamber and incubated for 3 days at $28^{\circ} \mathrm{C}$ with a 12 -h photoperiod. We investigated the development of CLS disease by means of a previously reported scale of 0 to 10 (Ishii et al. 2007). The disease index and disease suppression were calculated by the following formulas:

Disease index $=$

( $\sum[$ The number of diseased leaves in each index $x$ disease index $] /[$ The number of leaves investigated $\times 10]) \times 100$

Disease suppression $(\%)=$

(The DI of water control-The DI of treatment)/

The DI of water control $\times 100$

Resistance-risk assessment. Mutants resistant to isopyrazam, mefentrifluconazole, and their compound mixtures were generated by UV mutagenesis and fungicide selection methods previously reported, with some modifications (Zhai et al. 2014). The optimal proportion of the two-component mixture of isopyrazam and mefentrifluconazole (iso \& mef) was determined to be $1: 1$; this proportion was chosen for resistance risk assessment, and the following experiments were conducted with three independent repeats.

Mycelial discs were cut from the margin of an actively growing colony and then inoculated on PDA plates amended with $100 \mu \mathrm{g} / \mathrm{ml}$ isopyrazam and $100 \mu \mathrm{g} / \mathrm{ml}$ mefentrifluconazole. The uncapped plates were

Table 1. Cotoxicity coefficient (CTC) and synergistic ratio (SR) of binary mixtures of isopyrazam (Iso) and mefentrifluconazole (Mef) against Corynespora cassiicola $^{\mathrm{a}}$

\begin{tabular}{lccccc}
\hline Volume ratio (Iso:Mef) & EC $_{\mathbf{5 0}}(\boldsymbol{\mu} \mathbf{g} / \mathbf{m l})$ & CTC & Evaluation by CTC & SR & Evaluation by SR \\
\hline $1: 9$ & 6.47 & 110.33 & Addition & 1.10 & Addition \\
$1: 4$ & 5.73 & 83.76 & Addition & 0.84 & Addition \\
$3: 7$ & 3.37 & 107.24 & Addition & 1.07 & Addition \\
$2: 3$ & 2.70 & 107.34 & Addition & 1.07 & Addition \\
$1: 1$ & 0.74 & 325.17 & Synergism & 3.25 & Synergism \\
$3: 2$ & 3.28 & 63.31 & Antagonism & 0.63 & Addition \\
$7: 3$ & 4.47 & 40.61 & Antagonism & 0.41 & Antagonism \\
$4: 1$ & 5.88 & 27.49 & Antagonism & 0.27 & Antagonism \\
$9: 1$ & 6.54 & 22.25 & Antagonism & 0.22 & Antagonism \\
\hline
\end{tabular}

${ }^{a}$ C. cassiicola isolate $\mathrm{JN}-1$ was selected from our isolate collection to determine the sensitivity to the different binary mixtures of isopyrazam and mefentrifluconazole. 
exposed to UV light $(P=30 \mathrm{~W}, \lambda=254 \mathrm{~nm})$ at a vertical distance of $20 \mathrm{~cm}$ for $3 \mathrm{~min}$ and immediately placed in the dark for 5 to 7 days. All colonies that grew normally were considered suspected resistant mutants and needed to be recultured. Only the isolates that could once again grow well on the fungicide-containing plates were labeled as resistant mutants. Each isolate was grown on 10 plates, and each plate had four agar plugs.

For the fungicide selection method, mycelial plugs from a 5-dayold culture of $C$. cassiicola were transferred to new PDA plates supplemented with the tested fungicides. After 5 to 7 days of cultivation at $25^{\circ} \mathrm{C}$, the relatively fast-growing isolate was retransferred to a plate containing the test fungicides at higher concentrations. The above process was executed five times consecutively with the concentration of fungicides increasing sequentially $(50,100,150,200$, $250 \mu \mathrm{g} / \mathrm{ml}$ ), and the $\mathrm{EC}_{50}$ values were determined every generation. There were 10 plates per isolate and four mycelial plugs per plate.

Statistical analysis. In the present study, the $\mathrm{EC}_{50}$ value of each isolate was obtained using linear regression analysis by the software package DPS (China, version 13.5), and other data analysis was conducted by SPSS (IBM, USA, version 19.0). Pearson's $\chi^{2}$ test was performed to verify the virulence regression equation. Student's $t$ test was used to compare the mean between treatments in the in vitro fungicide sensitivity assays. To detect evident differences between treatments in the in vivo experiments, one-way analysis of variance (ANOVA) was used. Normality was assessed by the Shapiro-Wilk test before ANOVA, and data from our study met the assumptions of homogeneity of variance according to the Brown-Forsythe test. Fisher's least significant difference (LSD) test at $P<0.05$ was performed to compare means.

\section{Results}

Isopyrazam and mefentrifluconazole inhibited the mycelium growth, spore germination, and germ tube elongation of $C$. cassiicola. There was no significant difference $(P>0.05)$ in the inhibitory effect of each isopyrazam or mefentrifluconazole concentration on the same isolate; therefore, the inhibition rates of three independent replicates were pooled for subsequent analysis. With the increase of the concentration, the inhibition rates of isopyrazam and mefentrifluconazole against the three life stages of $C$. cassiicola were also gradually enhanced. In several life stages, isopyrazam was more effective in inhibiting spore germination and germ tube elongation (Fig. 2A-C). In contrast, the mycelial growth and spore germination of $C$. cassiicola were less sensitive to mefentrifluconazole (Fig. 2D-F). There was a significant difference $(P<0.05)$ in the sensitivity to isopyrazam and mefentrifluconazole at each life stage of $C$. cassiicola, and the mean $\mathrm{EC}_{50}$ values of isopyrazam to the pathogen were lower than that of mefentrifluconazole (Fig. 2G-I).

The optimal proportion of isopyrazam and mefentrifluconazole binary mixtures. Among the nine binary mixtures measured, the SRs were all greater than 0.5 when the proportion of isopyrazam
A

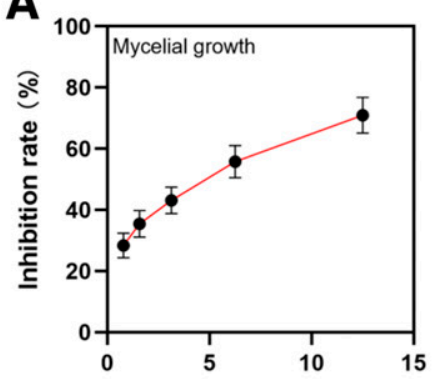

Isopyrazam concentration $(\mu \mathrm{g} / \mathrm{mL})$

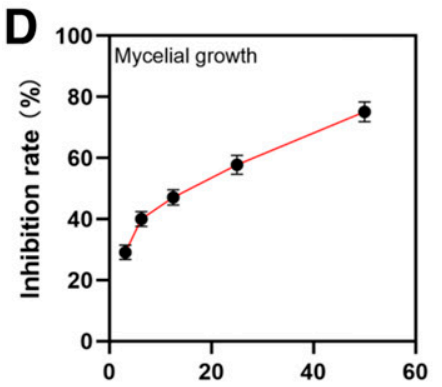

Mefentrifluconazole concentration $(\mu \mathrm{g} / \mathrm{mL})$
B

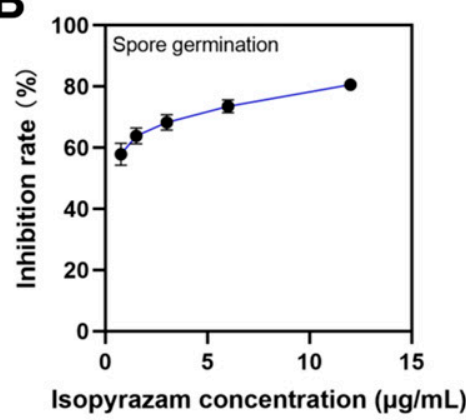

E

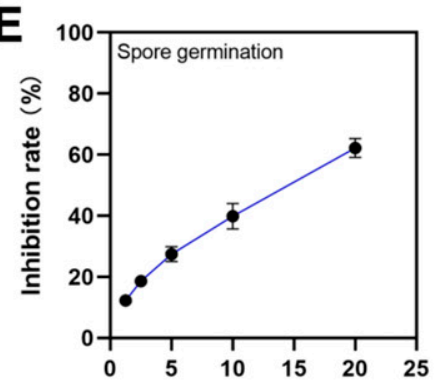

Mefentrifluconazole concentration $(\mu \mathrm{g} / \mathrm{mL})$

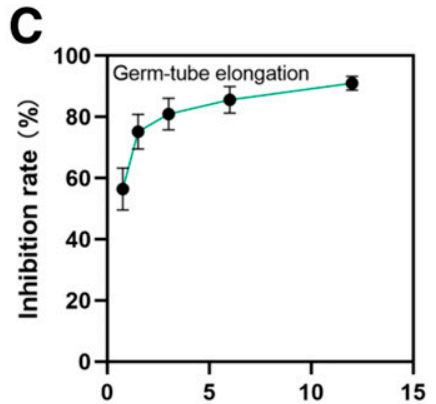

Isopyrazam concentration $(\mu \mathrm{g} / \mathrm{mL})$

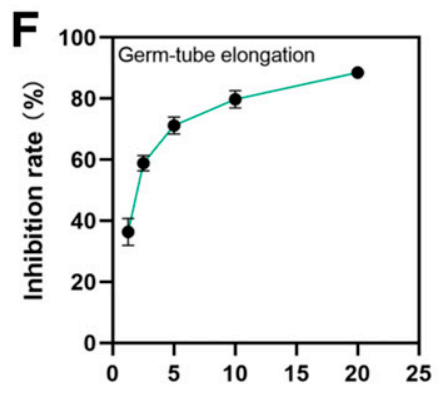

Mefentrifluconazole concentration $(\mu \mathrm{g} / \mathrm{mL})$
G

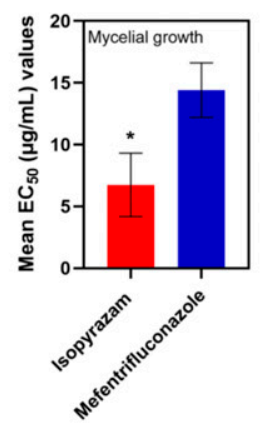

H

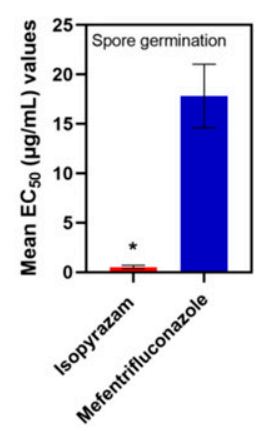

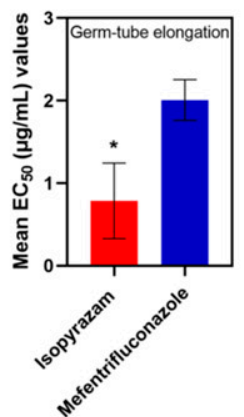

Fig. 2. In vitro sensitivity of Corynespora cassiicola to isopyrazam and mefentrifluconazole based on mycelial growth (A, D), spore germination (B, E), and germ tube elongation (C, F). Mean $\mathrm{EC}_{50}$ values of isopyrazam and mefentrifluconazole to mycelial growth $(\mathbf{G})$, spore germination $(\mathbf{H})$, and germ tube elongation (I) of $10 \mathrm{C}$. cassiicola isolates collected from greenhouses of Shandong, China. Error bars represent mean $\pm \mathrm{SD}\left(n=3 ;{ }^{*} P<0.05\right.$, Student's $t$ test). 
was $\leq 60 \%$. According to the $\mathrm{CTC}$ analysis, as long as the proportion of isopyrazam does not exceed $50 \%$, additive or synergistic effects can be observed. In numerical terms, the CTC was equal to the SR multiplied by 100 , and the optimal ratio determined by both methods was 1:1 (isopyrazam/mefentrifluconazole) (Table 1). After combining the arcsine conversion value of the percent of isopyrazam in the mixture and its corresponding CTC value, the mathematical model was fitted as $\mathrm{Y}=-0.14 \mathrm{X}^{2}+10.40 \mathrm{X}-57.79$. The theoretical maximum value of CTC was 140.38 ( $\mathrm{X}=38.22, \mathrm{~K}=38.27 \%$ ), which was far below the maximum CTC value actually observed (CTC = $325.17, X=45.00, K=50.00 \%$ ) (Fig. 1).

Isopyrazam and mefentrifluconazole at the optimum mixture enhanced the in vitro and in vivo bioactivity against $C$. cassiicola. The $\mathrm{EC}_{50}$ values of 10 tested $C$. cassiicola isolates ranged from 0.19 to $5.33 \mu \mathrm{g} / \mathrm{ml}$ after treatment with iso \& mef (1:1), with corresponding CTCs with a minimum of 208.25, which were all considered synergy (Table 2).

After protective and curative applications, the disease index of CLS on potted cucumber seedlings was significantly reduced $(P<$ 0.05 ) by all fungicide treatments (Fig. 3A). For protective activity, iso \& mef (1:1) at $100 \mu \mathrm{g}$ a.i./ml achieved the best disease

Table 2. Sensitivity of Corynespora cassiicola isolates to the combination of isopyrazam and mefentrifluconazole at 1:1 and their synergistic effect

\begin{tabular}{lccc}
\hline Isolate code & EC $_{\mathbf{5 0}}(\boldsymbol{\mu} \mathbf{g} / \mathbf{m l})$ & Cotoxicity coefficient $(\mathbf{C T C})$ & Evaluation \\
\hline LY-1 & 0.76 & 393.69 & Synergism \\
LY-2 & 1.32 & 381.24 & Synergism \\
WF-1 & 0.64 & 248.91 & Synergism \\
WF-2 & 0.65 & 258.20 & Synergism \\
LW-1 & 0.98 & 208.25 & Synergism \\
DZ-1 & 0.90 & 370.34 & Synergism \\
JN-1 & 0.74 & 325.17 & Synergism \\
TA-1 & 5.33 & 314.23 & Synergism \\
LC-1 & 1.75 & 239.43 & Synergism \\
ZB-1 & 0.19 & 233.21 & Synergism \\
\hline
\end{tabular}

suppression of CLS on potted cucumbers (42.12\%), while mefentrifluconazole at $100 \mu \mathrm{g}$ a.i./ml showed the worst disease suppression $(24.65 \%)$. In the in vivo curative activity experiment, iso \& mef (1:1) had the best inhibition of the disease $(42.84 \%)$, followed by mefentrifluconazole (37.48\%) and isopyrazam (33.90\%) (Fig. 3B).

Resistance-risk assessment. The sensitivity of $C$. cassiicola isolates to various fungicides changed in both the UV mutagenesis experiment and the fungicide selection experiment (Fig. 4). After UV treatment, the sensitivity of the mutant isolates to isopyrazam, mefentrifluconazole, and iso \& mef (1:1) decreased, and the mean $\mathrm{EC}_{50}$ values increased from 3.19 to $28.89,14.41$ to 19.72 , and 1.36 to $19.34 \mu \mathrm{g} / \mathrm{ml}$, respectively (Fig. 4A). The number of isolates with reduced sensitivity to mefentrifluconazole was the most, while the number of isolates with reduced sensitivity to iso \& mef (1:1) was the least (Fig. 4B). In fungicide-selection assays, although the inhibition effect of each fungicide on the isolates induced by five successive generations decreased, no significant difference was found in the sensitivity of these isolates to isopyrazam, mefentrifluconazole, and iso \& mef (1:1) (Fig. 4C). After the fifth fungicide treatment, there were four isolates with reduced sensitivity to isopyrazam, six isolates to mefentrifluconazole, and three isolates to iso \& mef (1:1) (Fig. 4D).

\section{Discussion}

In vitro fungicide sensitivity experiments showed that there were differences in the sensitivity of $C$. cassiicola to isopyrazam or mefentrifluconazole at different life stages, and the inhibition effects of isopyrazam on mycelium growth, spore germination, and germ tube elongation were stronger than that of mefentrifluconazole (Fig. 2). The bioactivity of isopyrazam against $C$. cassiicola examined in our work was slightly different from published data (He et al. 2018). In the current study, isolates obtained from greenhouses in eight different cities of Shandong, China, were tested, while in the previous report, only four isolates were collected from greenhouses in Tai'an, Shandong. We had reason to attribute the difference between the two studies to the sampling regions and the number of isolates, and
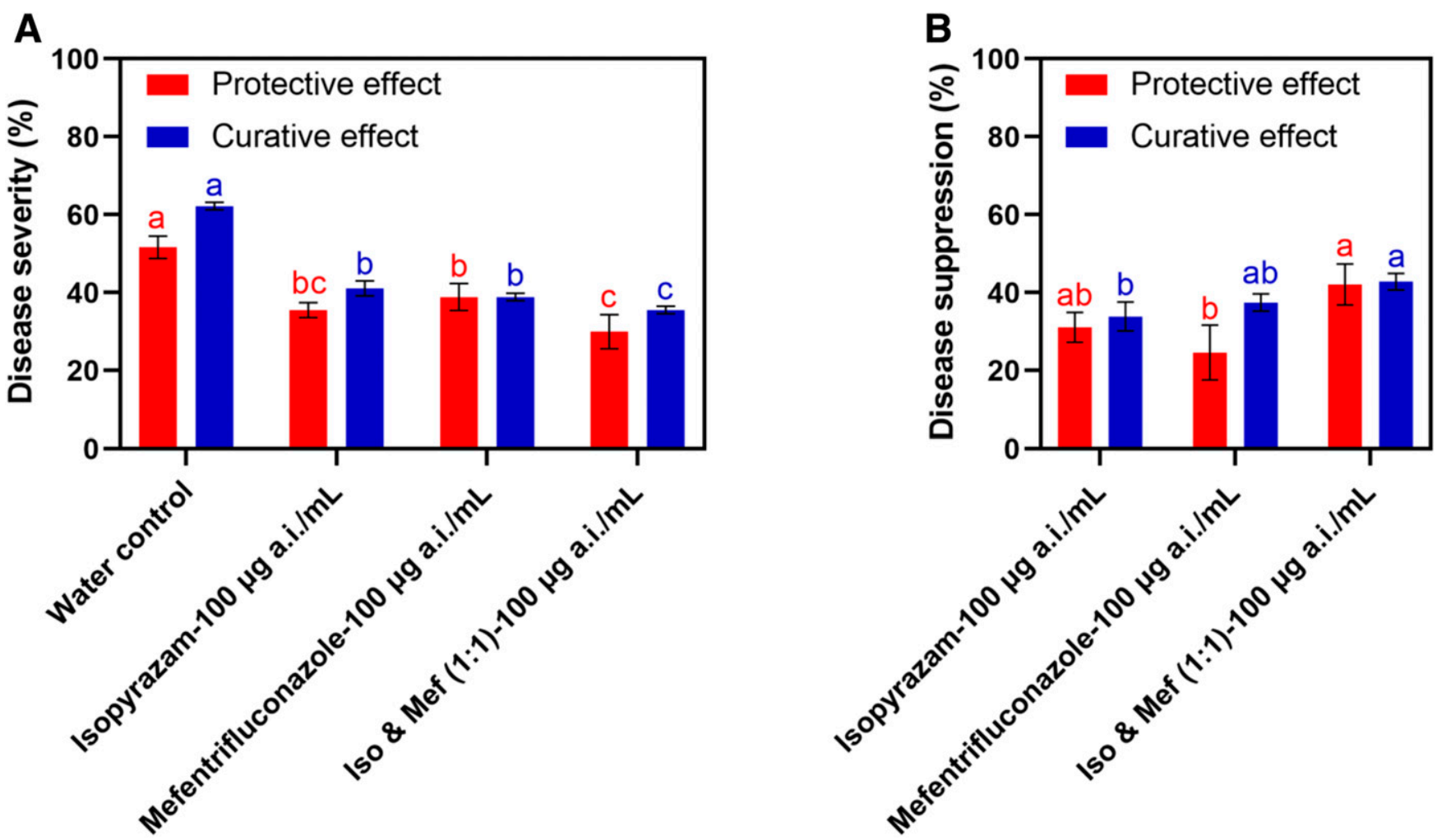

Fig. 3. Disease index (A) and disease suppression (B) of isopyrazam, mefentrifluconazole, and their combination at 1:1 against Corynespora leaf spot for in vivo inoculation tests conducted in the greenhouse. All fungicides were applied at $100 \mu \mathrm{g}$ a.i. $/ \mathrm{ml}$. Iso $=$ isopyrazam, Mef $=$ mefentrifluconazole. Error bars represent mean $\pm \mathrm{SD}(n=3)$. Different letters correspond to significant differences based on ANOVA $(P<0.05)$. 
there was no doubt that a larger sampling should be conducted for further analysis. A previous study suggested that the inhibitory effect of isopyrazam on spore germination of $B$. cinerea was far greater than that on mycelial growth (Song et al. 2016). Only $0.06 \mu \mathrm{g} / \mathrm{ml}$ isopyrazam can almost completely inhibit spore germination, but to achieve this effect, the inhibitory concentration of mycelial growth should be adjusted to at least $40 \mu \mathrm{g} / \mathrm{ml}$ (Song et al. 2016). This result can be attributed to the fact that the SDHI fungicide isopyrazam exerted the greatest influence on spore germination, an energy-consuming stage. Coincidentally, germ tube elongation of $C$. cassiicola was the most sensitive to the triazole fungicides difenoconazole, tebuconazole, and epoxiconazole, while spore germination was the least sensitive stage (He et al. 2018). We believe that this is mainly because these compounds act by destroying the cell membrane of the pathogen.

The CTC method was employed to verify the synergistic effect of mixed preparations against red rot caused by Fusarium moniliforme with seven different fungicides. The results showed that when difenoconazole and carbendazim were combined in a 1:1 ratio, the $\mathrm{EC}_{50}$ value was significantly lower than that of a single fungicide, with a CTC of 286, which had the greatest synergistic effect (Pan et al. 2007). Two hypotheses about the mechanism of the improved activity of pesticide compounds have been proposed: the first mechanism suggests that the components of the compound acted on different stages of the disease cycle, and the synergistic effect of several factors was higher than that of a single factor; the second mechanism was closely related to the absorption, diffusion, and degradation of the components of the mixture, that is, one component of the mixture may increase the absorption and translocation of another component (Gisi et al. 1985). Determining synergistic combinations and maximal synergistic ratios has always been important for pesticide compound research. Many methods have been developed for this purpose, such as Bliss's method, Sakai's method, the CTC method, the cotoxic factor method, and the toxic equivalency method (Bliss 1939; Harris and Chambers 1973; Mansour et al. 1966; Sakai 1960; Sun and Johnson 1960). In China, the CTC method and SR are commonly used to determine the joint virulence of compound fungicides (MAPRC 2006). In fact, these two methods involve different expression formulas from the same calculation, and different judgment standards give different synergistic effect results. Furthermore, the combination of a mathematical model and the CTC method has been suggested to determine the optimum proportion of binary mixtures (Zhang et al. 2006). Unexpectedly, there was a large negative deviation between the fitting curve and the measured results, and the highest CTC value derived by the mathematical model was much lower than that determined in our experiment. We attributed this difference mainly to the fact that this fitted data model is not sufficient to truly and accurately predict the optimal ratio of the two fungicides. In our work, we finally determined the optimum proportion according to the CTC method. It should be noted that the sensitivity of the isolate used to assess synergism needs to be stable throughout the experiment. The sensitivity of the isolate with quantitative resistance gradually decreased, which affected the final CTC or SR value, resulting in inaccurate evaluation results.

It is essential that the fungicides used for modern agricultural production have both preventative and curative effects (Miao et al. 2016); nevertheless, most studies focus on the protectiveness or curativeness of fungicides. In greenhouse pot experiments, isopyrazam, mefentrifluconazole, and iso \& mef (1:1) effectively reduced the disease index of CLS on potted cucumber before and after inoculation with $C$. cassiicola. Iso \& mef (1:1) showed better protective activity than mefentrifluconazole, and better curative activity than isopyrazam.

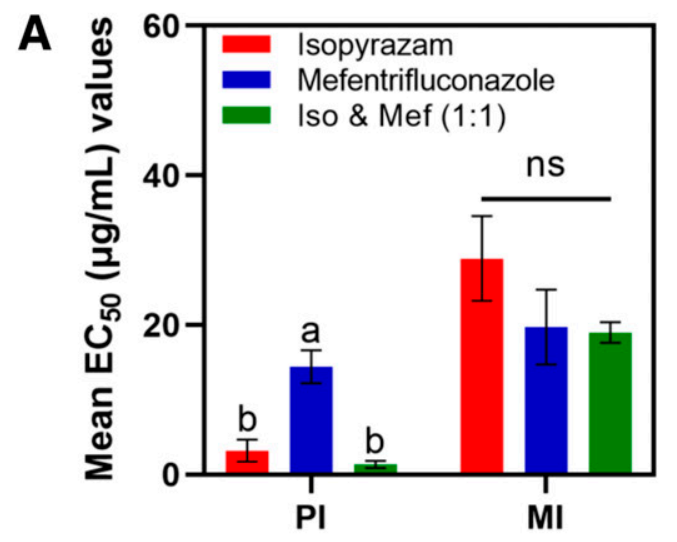

C

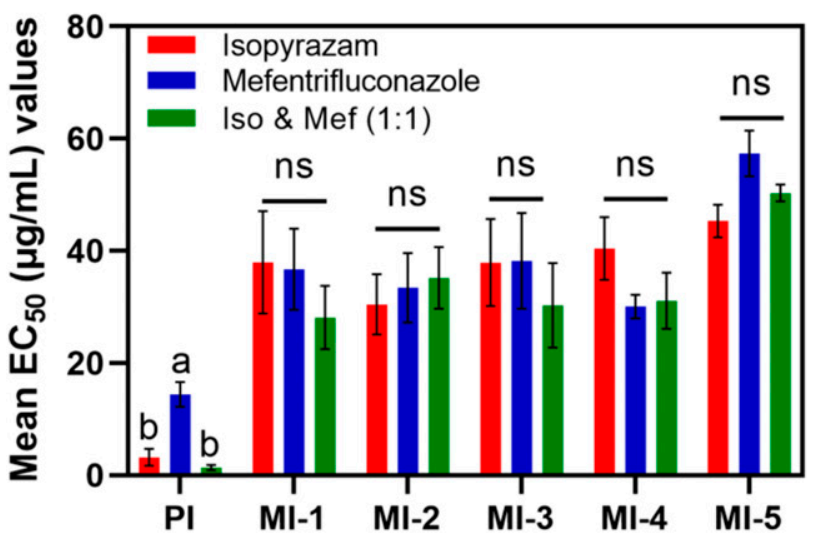

B

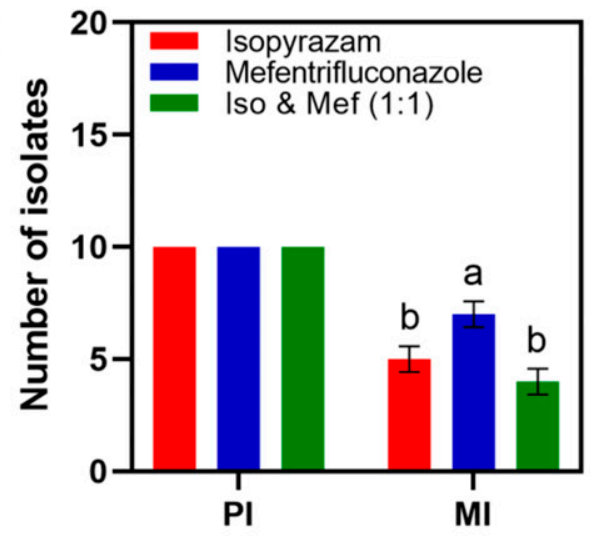

D

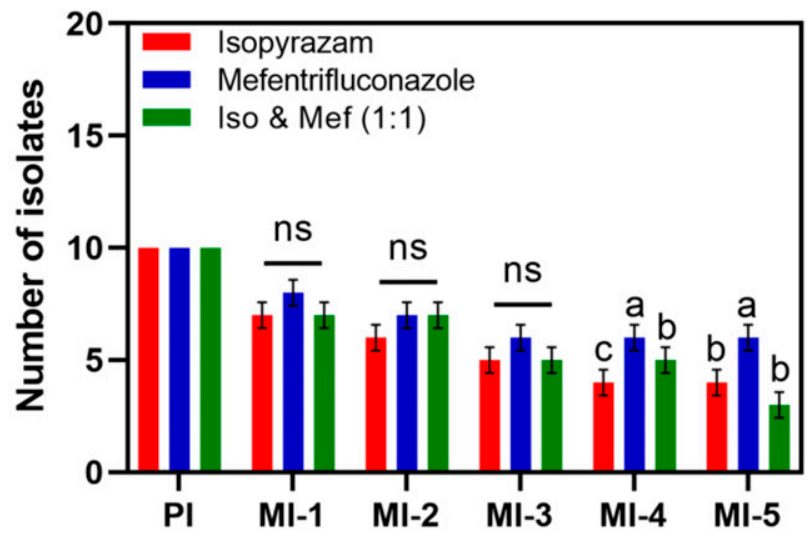

Fig. 4. In vitro sensitivity changes and the number of resistant mutants of Corynespora cassiicola isolates to isopyrazam, mefentrifluconazole, and their combination at $1: 1$ in UV mutagenesis assays $(\mathbf{A}, \mathbf{B})$ and fungicide-selection assays with five consecutive generations of resistance induction $(\mathbf{C}, \mathbf{D})$. $\mathrm{PI}=\mathrm{parental}$ isolates, $\mathrm{MI}=\mathrm{mutant}$ isolates, Iso = isopyrazam, Mef $=$ mefentrifluconazole, $\mathrm{ns}=$ not significant. Error bars represent mean $\pm \mathrm{SD}(n=3)$. Different letters correspond to significant differences based on ANOVA $(P<0.05)$. 
Currently, many fungicides with different MOAs have been mixed to manage CLS in China (Ma et al. 2018). However, whether the combination of fungicides can delay and prevent the occurrence of resistance and to what extent the fungicides work are still unclear. In this study, both the UV mutagenesis method and fungicideselection method were employed to assess the resistance risk of $C$. cassiicola isolates to isopyrazam, mefentrifluconazole, and their combination. In two different resistance risk assessment tests, there was no significant difference between the sensitivity change of isolates to isopyrazam, mefentrifluconazole, and iso \& mef (1:1). After $\mathrm{UV}$ and continuous fungicide induction, the number of isolates with reduced sensitivity to isopyrazam was similar to the number of isolates with reduced sensitivity to iso \& mef (1:1), which was lower than that to mefentrifluconazole. In summary, compared with a single fungicide, the combination of isopyrazam and mefentrifluconazole did not exert a great influence on the sensitivity change of $C$. cassiicola. We speculated that the reasons for this phenomenon might be as follows: (1) the resistance induction method may not be appropriate, which led to the failure to simulate the conditions for the field resistance occurrence; (2) the two fungicides selected in this study did not have an obvious effect on delaying resistance as we expected, so we should screen other fungicides with different MOAs for further verification.

Due to their single-site specificity, SDHI fungicides are considered easy to develop resistance (Fraaije et al. 2012). FRAC proposed that these chemicals were at a medium-high risk of developing resistance. Triazole fungicides, which are demethylation inhibitors, were also considered to be at medium risk. In our work, all the current risk assessment results were based entirely on laboratory tests. Considering the complex conditions in the field, a more detailed and systematic study should be performed to assess the effects of fungicides on pathogen resistance.

Currently, chemical treatment has been widely used in agricultural practice to protect crops from plant pathogens (Hunter et al. 2018). Due to the long-term unscientific abuse of agricultural chemicals, the problem of resistance has become increasingly prominent, leading to a decrease in the disease suppression and an increase in the shortage of highly effective fungicides (Hollomon 2015). Our experimental data provide support for applying these two fungicides individually or in combination on cucumber to manage CLS.

\section{Literature Cited}

Bliss, C. I. 1939. The toxicity of poisons applied jointly. Ann. Appl. Biol. 26: 585-615.

Date, H., Kataoka, E., Tanina, K., Sasaki, S., Inoue, K., Nasu, H., and Kasuyama, S. 2004. Sensitivity of Corynespora cassicola, causal agent of Corynespora leaf spot of cucumber, to thiophanate-methyl, diethofencarb and azoxystrobin. Jpn. J. Phytopathol. 70:10-13.

de Albuquerque, J. R. T., Silva, S., de Oliveira, F. A., de Paiva, E. P., Araújo, E. B. G., and Souto, L. S. 2016. Initial growth and tolerance of under salt stress cucumber cultivars. Rev. Bras. Agric. Irrig. 10:486-495.

De Wolf, E. D., and Isard, S. A. 2007. Disease cycle approach to plant disease prediction. Annu. Rev. Phytopathol. 45:203-220.

Dixon, L. J., Schlub, R. L., Pernezny, K., and Datnoff, L. E. 2009. Host specialization and phylogenetic diversity of Corynespora cassiicola. Phytopathology 99:1015-1027.

Fraaije, B. A., Bayon, C., Atkins, S., Cools, H. J., Lucas, J. A., and Fraaije, M. W. 2012. Risk assessment studies on succinate dehydrogenase inhibitors, the new weapons in the battle to control Septoria leaf blotch in wheat. Mol. Plant Pathol. 13:263-275.

FRAC. 2019. Pathogen Risk List 2019. Fungicide Resistance Action Committee, CropLife International, Brussels, Belgium. https://www.frac.info/docs/defaultsource/publications/pathogen-risk/frac-pathogen-list-2019.pdf.

Gisi, U., Binder, H., and Rimbach, E. 1985. Synergistic interactions of fungicides with different modes of action. Trans. Br. Mycol. Soc. 85:299-306.

Gudmestad, N. C., Arabiat, S., and Pasche, J. S. 2013. Prevalence and impact of SDHI fungicide resistance in Alternaria solani. Phytopathology 97:952-960.

Harp, T. L., Godwin, J. R., Scalliet, G., Walter, H., Stalker, A. D., Bartlett, D. W., and Ranner, D. J. 2011. Isopyrazam, a new generation cereal fungicide. Asp. Appl. Biol. 106:113-120.

Harris, F. A., and Chambers, H. W. 1973. Interaction of insecticidal mixtures in tobacco budworm. J. Econ. Entomol. 66:517-518.

He, L. F., Li, X. X., Zhu, J. M., Mu, W., and Liu, F. 2018. Comparison of toxicity properties of different types of fungicides against Corynespora cassiicola on cucumber. Chin. J. Pestic. Sci. 20:25-32.
Hollomon, D. W. 2015. Fungicide resistance: facing the challenge. Plant Prot. Sci. 51:170-176.

Hunter, S., Williams, N., McDougal, R., Scott, P., and Garbelotto, M. 2018 Evidence for rapid adaptive evolution of tolerance to chemical treatments in Phytophthora species and its practical implications. PLoS One 13: e0208961.

Ishii, H., Miyamoto, T., Ushio, S., and Kakishima, M. 2011. Lack of crossresistance to a novel succinate dehydrogenase inhibitor, fluopyram, in highly boscalid-resistant isolates of Corynespora cassiicola and Podosphaera xanthii. Pest Manag. Sci. 67:474-482.

Ishii, H., Yano, K., Date, H., Furuta, A., Sagehashi, Y., Yamaguchi, T., Sugiyama, T., Nishimura, K., and Hasama, W. 2007. Molecular characterization and diagnosis of QoI resistance in cucumber and eggplant fungal pathogens. Phytopathology 97:1458-1466.

Kingsland, G. C. 1986. Pathogenicity and epidemiology of Corynespora cassiicola in the Republic of Seychelles. Int. J. Pest Manage. 32:283-287.

Koller, W., and Scheinpflug, H. 1987. Fungal resistance to sterol biosynthesis inhibitors: a new challenge. Plant Dis. 71:1066-1074.

Li, B. J., Gao, W., Shi, Y. X., and Xie, X. W. 2012. Progress in researches on Corynespora leaf spot. Acta Phytophylac. Sin. 39:171-176.

Luo, F. C., Yuan, Q. H., and Wang, Y. 2016. Toxicity test of different fungicides and compound formulations to alfalfa rust. Acta Agrestia Sin. 24:165-170

Ma, D. C., Zhu, J. M., Jiang, J. G., Zhao, Y. H., Li, B. X., Mu, W., and Liu, F. 2018. Evaluation of bioactivity and control efficacy of tetramycin against Corynespora cassiicola. Pestic. Biochem. Physiol. 152:106-113.

Mansour, N. A., Eldefrawi, M. E., Toppozada, A., and Zeid, M. 1966. Toxicological studies on the Egyptian cotton leaf worm, Prodenia litura. VI. Potentiation and antagonism of organophosphorus and carbamate insecticides. J. Econ. Entomol. 59:307-311.

MAPRC. 2006. Pesticides guidelines for laboratory bioactivity tests-Part 6 Determining combined action of fungicide mixtures. Ministry of Agriculture of People's Republic of China. China Standards Press, Beijing.

Miao, J. Q., Cai, M., Dong, X., Liu, L., Lin, D., Zhang, C., Pang, Z. L., and Liu, X. L. 2016. Resistance assessment for oxathiapiprolin in Phytophthora capsici and the detection of a point mutation (G769W) in PcORP1 that confers resistance. Front. Microbiol. 7:615.

Miyamoto, T., Ishii, H., Seko, T., Kobori, S., and Tomita, Y. 2009. Occurrence of Corynespora cassiicola isolates resistant to boscalid on cucumber in Ibaraki Prefecture. Japan. Plant Pathol. 58:1144-1151.

Miyamoto, T., Ishii, H., Stammler, G., Koch, A., Ogawara, T., Tomita, Y., Fountaine, J. M., Ushio, S., Seko, T., and Kobori, S. 2010. Distribution and molecular characterization of Corynespora cassiicola isolates resistant to boscalid. Plant Pathol. 59:873-881.

Pan, Y. M., Gao, Z. M., Wang, J. X., Cao, J., and Wang, T. 2007. Studies on cotoxicity of the mixed preparations of several fungicides to Fusarium moniliforme. Cotton Sci. 19:93-97.

Sakai, S. 1960. Insect toxicological studies on the joint toxic action of insecticides. J. Emerg. Med. 34:441-450.

Song, Y. Y., Zhang, Z. Q., Chen, L. L., He, L. M., Lu, H. B., Ren, Y. P., Mu, W. and Liu, F. 2016. Baseline sensitivity of Botrytis cinerea to the succinate dehydrogenase inhibitor isopyrazam and efficacy of this fungicide. Plant Dis. 100:1314-1320.

Sun, F. L., Bao, B. F., Ma, L. F., Chen, A. L., and Duan, X. F. 2012. Mouldresistance of bamboo treated with the compound of chitosan-copper complex and organic fungicides. J. Wood Sci. 58:51-56.

Sun, Y. P., and Johnson, E. 1960. Analysis of joint action of insecticides against house flies. J. Econ. Entomol. 53:887-892.

Tang, Q. Y., and Zhang, C. X. 2012. Data processing system (DPS) software with experimental design, statistical analysis and data mining developed for use in entomological research. Insect Sci. 20:254-260.

Wadley, F. M. 1967. Experimental Statistics in Entomology. Graduate School Press, Washington, DC, U.S.A

Yu, S. J., Wang, M. Y., Tian, F., Zhao, W. G., Bian, Q., and Li, B. J. 2014. Progress in research on control of cucumber Corynespora leaf spot and fungicide resistance. Agrochemicals 53:7-11+33.

Zhai, M. T., Wang, K. Y., Xu, H., Tang, J. F., and Liu, J. 2014. Induction and characteristics of Phytophthora capsici isolates resistant to fluopicolide. Acta Phytopathologica Sin. 44:88-96.

Zhang, W., Deng, X. P., Wang, J. J., He, L., and Wu, C. X. 2006. Use of a mathematical model in screening optimal proportions of two-component mixtures. Agrochemicals 45:316-319.

Zhang, Y. J., Liu, D., Ma, B. Z., Zhou, X. Y., and Miao, D. 2014. Occurrence and identification of cucumber target spot in Heilongjiang Province. J. Northeas Agric. Univ. 45:1-7.

Zhang, Z. G., Zhang, K. Z., Zheng, X. D., He, X. H., Kong, L. L., and Lin, N. 2019. A review of synthetic methods of mefentrifluconazole. Modern Agrochemicals 18:24-26+34 